\section{Nuove terapie oncologiche e "giusto" prezzo}

Dear Editor,

In Europa, a differenza degli Stati Uniti, il Diritto alla Salute è incluso tra i diritti costituzionali e, come conseguenza, nella grande maggioranza dei casi, specie per terapie di valore, il costo della terapia stessa, farmacologica e no, viene sostenuto dai Servizi Sanitari Nazionali (SSN). È per tale motivo che quasi tutti i Paesi europei, compresi i ventotto Paesi dell'Unione Europea, prevedono dei sistemi di definizione del "prezzo rimborsato" di un farmaco, spesso tramite un processo strutturato di negoziazione.

Storicamente tale negoziazione del prezzo dei farmaci si è basata su due vettori fondamentali: da una parte quello legato alla politica economico-industriale e dall'altra quello legato al beneficio clinico per il paziente. Nel secolo scorso le negoziazioni, strettamente riservate, dell'Autorità inglese con le Aziende Farmaceutiche e quelle più o meno riservate di molte altre Autorità europee, compresa l'Italia, tenevano conto degli investimenti produttivi e degli investimenti in R\&D nel Paese, nonché di ricadute sul livello di occupazione garantito o promesso. Questa metodologia, sebbene fondata su considerazioni comprensibili e anche in parte condivisibili, è stata oggetto delle critiche di chi riteneva che la politica industriale dovesse utilizzare strumenti diversi quali, ad esempio, incentivi all'attività di R\&D e altre forme di sostegno, sganciate però dal prezzo del farmaco pagato dai SSN.

L'avanzare dell'Unione Europea e del conseguente Mercato Unico, con la promessa della libera circolazione delle merci all'interno dell'Unione, ha spostato l'equilibrio in favore di quanti sostenevano e sostengono che il costo della terapia, e in generale il costo di qualunque intervento sanitario, dovrebbe basarsi sul beneficio clinico per i pazienti e quindi dovrebbe essere molto più simile in tutta l'Unione, pur in presenza di fondi di finanziamento nazionali gestiti dai rispettivi Servizi Sanitari.

Queste mie considerazioni prendono spunto dall'articolo di Martone e colleghi, pubblicato nell'ultimo numero di GRHTA [2014;1(2):31-43], sulla valutazione di costo-efficacia dei nuovi farmaci oncologici immessi sul mercato italiano. Questo articolo si è posto l'obiettivo di fotografare la situazione più recente di una classe di farmaci di particolare rilievo, sia per la loro crescente importanza clinica sia per la rilevanza della spesa relativa. Un passo importante, alla luce dell'orientamento moderno basato principalmente sul vettore legato al beneficio clinico, che dovrebbe proseguire con l'esame comparato dei rapporti di costo efficacia all'interno di una stessa classe terapeutica, anche negli altri Paesi europei, quantomeno i 28 dell'Unione.

Nell'articolo si discute la questione delle "soglie" di accettabilità. Un argomento delicato e sul quale è al momento difficile trovare un consenso. Le soglie potrebbero anche dipendere dalla percentuale di aumento della sopravvivenza nella specifica patologia, dalla rarità della patologia stessa e, soprattutto, risentire della ricchezza del singolo Paese. Molti i punti in discussione: ad esempio se debba essere applicata la valutazione HTA (e con quali soglie) per i farmaci orfa$\mathrm{ni}$ - cioè quei farmaci indicati per condizioni cliniche rare e gravi - quando non esista ancora un valido trattamento, o che comunque presentino un vantaggio clinico significativo rispetto all'esistente, valutato dal COMP di EMA. Di fatto, per la maggior parte dei farmaci orfani, le Agenzie dei singoli Paesi dell'Unione Europea hanno deciso per il rimborso anche in caso di costi per singolo paziente molto elevati (superiori a 100 mila euro/anno), tenuto conto di un "budget impact" comunque contenuto.

Sappiamo poi che l'ammissione di un nuovo farmaco al beneficio della rimborsabilità dipende anche da altri fattori, diversi dal rapporto di costo-efficacia, quali l'approccio culturale ed etico del Paese, o anche dalla forza dell'Associazione locale dei pazienti affetti da quella determinata patologia, come opportunamente commentato dagli Autori dell'articolo sopra citato. Inoltre, Paesi di tradizione prevalentemente protestante, quali quelli del Nord Europa, pur più "ricchi", spesso hanno un atteggiamento più severo di altri Paesi del Sud Europa con anche una tendenza, nella pratica, a discriminare, a volte, tra i pazienti giovani e quelli anziani piuttosto che tra quelli in migliore situazione clinica rispetto a quelli alle ultime fasi della malattia. Rimane comunque difficile negare un trattamento quando si sia in presenza di una malattia molto grave e si disponga di dati di aumento di sopravvivenza, seppure quantitativamente limitata, basati sull'evidenza clinica. Questa considerazione è ben presente nei commenti conclusivi degli Autori e non può che essere sottoscritta.

L'augurio è che l'articolo in oggetto stimoli la comunità scientifica che si occupa di questi argomenti ad allargare il campo di rilevazione e analisi critica a tutto il panorama europeo, in modo da comprendere come le diverse Autorità regolatorie si stiano orientando, se verso sistemi di ValueBased Pricing (VBP) o verso meccanismi di Payment-by-Results $(\mathrm{PbR})$, oppure verso entrambi. Fondamentale anche capire quali siano i rapporti tra queste due alternative. II $\mathrm{PbR}$, ad esempio, è ampiamente usato in Italia in questi ultimi anni per le terapie oncologiche, e potrebbe essere un modello esportabile negli altri Paesi visti i suoi potenziali vantaggi per il SSN e per i pazienti, e in definitiva anche per le aziende farmaceutiche. Per essere efficace un modello di questo genere, in base al quale l'Azienda produttrice si impegna a restituire al SSN il costo della terapia sostenuto per i pazienti che non hanno avuto benefici, deve essere collegato a un Registro elettronico. Prima di poterlo esportare bisogna però che il modello dimostri di funzionare bene prima di tutto in Italia: a questo proposito siamo in attesa delle evidenze di funzionamento dei nuovi Registri AIFA.

Infine non va dimenticato che, per quanto vi siano terapie costose, gli unici farmaci che, al momento, rischiano di mettere 
in crisi il sistema sono quelli per l'epatite $\mathrm{C}$, che hanno un costo da "farmaco orfano" per una popolazione di pazienti che purtroppo in alcuni Paesi, tra i quali l'Italia, è molto estesa. D’altra parte queste nuove terapie promettono di ridurre fortemente il numero di pazienti che soffrono di questa patologia, con vantaggi importanti e risparmi di spesa complessiva nel medio periodo. In ogni caso, in presenza di un elevato rischio di utilizzo diffuso di questi medicinali - elemento che li differenzia ulteriormente dai farmaci orfani - un'accurata selezione dei pazienti e meccanismi di rigoroso controllo dell'appropriatezza, quale quello di un Registro AIFA applicato in modo vincolante, potrebbero contenere la spesa relativa.

Rimane poi sempre la considerazione più generale sugli equilibri della spesa sanitaria: la sostenibilità del SSN dovrebbe essere valutata in modo unitario e forse I'HTA è una metodologia che aiuterà a tenere conto di tutte le attività, non solo di quelle relative alle nuove terapie farmacologiche, contribuendo contemporaneamente alla tutela della salute e al buon uso delle risorse.

\section{Disclosures}

Financial support: None.

Conflict of interest: EB is an employee of Celgene Italia.

\section{Enrico Bosone}

Direttore Corporate Affairs and Market Access, Celgene srl, Milano - Italy

Indirizzo per la corrispondenza:

Enrico Bosone

Direttore Corporate Affairs and Market Access

Celgene srl

Via della Liberazione 13

20124 Milano, Italy

ebosone@celgene.com

Published online: December 19, 2014 